\title{
EXISTENCE OF SYMMETRIC SKEW BALANCED STARTERS FOR ODD PRIME POWERS
}

\author{
DING-ZHU DU AND F. K. HWANG
}

(Communicated by Thomas H. Brylawski)

\begin{abstract}
Strong starters and skew starters have been widely used in various combinatorial designs. In particular skew balanced starters and symmetric skew balanced starters are crucially used in the construction of completely balanced Howell rotations. Let $n=2^{m} k+1$ be an odd prime power where $m \geq 2$ and $k$ is an odd number. The existence of symmetric skew balanced starters for $G F(n)$ has been proved for $m \geq 2$ and $k \neq 1,3,9$. In this paper, we present a new approach which gives a uniform proof of the existence of symmetric skew balanced starters for all $m \geq 2$ and $k \geq 3$.
\end{abstract}

1. Introduction. Let $n$ be an odd prime power. A set of $p=(n-1) / 2$ pairs $\left(x_{1}, y_{1}\right), \ldots,\left(x_{p}, y_{p}\right)$ is called a skew balanced starter (SBS) if

(i) $\left\{x_{1}, y_{1}, \ldots, x_{p}, y_{p}\right\}=G F(n)-\{0\}$,

(ii) $\left\{ \pm\left(x_{i}-y_{i}\right): i=1, \ldots, p\right\}=G F(n)-\{0\}$,

(iii) $\left\{ \pm\left(x_{i}+y_{i}\right): i=1, \ldots, p\right\}=G F(n)-\{0\}$,

(iv) the multiset of differences $\left\{x_{i}-x_{j}: i, j=1, \ldots, p, i \neq j\right\}$ and the multiset of differences $\left\{y_{i}-y_{j}: j=1, \ldots, p, i \neq j\right\}$ together contain every nonzero element of $G F(n)$ the same number of times.

An SBS is called symmetric and denoted by SSBS if in addition:

(v) $\left\{x_{1}, \ldots, x_{p}\right\}=\left\{-x_{1}, \ldots,-x_{p}\right\}$ (which implies $\left\{y_{1}, \ldots, y_{p}\right\}=\left\{-y_{1}, \ldots\right.$, $\left.\left.-y_{p}\right\}\right)$.

Write $n=e k+1$ where $e=2^{m}$ and $k$ is odd. Note that condition (v) implies that $p$ must be even, hence $m \geq 2$. It is well known [3] that an SBS of order $n=2 k+1$ yields a complete balanced Howell rotation (also known as balanced Room square) of order $n+1$ and an SSBS of order $n=2^{m} k+1$ for $m \geq 2$ yields a complete balanced Howell rotation of order $2(n+1)$. Berlekamp and Hwang [1] gave a construction for SBS of order $n=2 k+1$ for all odd $k>1$. The construction of SSBS has been extensively studied in the literature (see [5] for a review). In particular, Du and Hwang [3] gave a construction which works for (i) $m=2$ and $k>1$, (ii) $m=3$ and $k>1$, (iii) $m \geq 4$ and $k>9 e^{3}$. Later, Yu and Hwang [7] showed that the construction of Du and Hwang works for all $m \geq 2$ and $k \geq 4 e$. They also conjectured that the construction works for all $m \geq 2$ and $k>1$. Recently, Yu and Hwang [8] proved the conjecture except for the two cases of $k=3$ and $k=9$. In this paper, we present an alternative approach to show the existence of SSBS including the two cases $k=3$ and $k=9$.

Received by the editors May 12, 1986 and, in revised form, July 6, 1987.

1980 Mathematics Subject Classification (1985 Revision). Primary 05B15; Secondary 05B10, $12 \mathrm{C} 20$.

Research of the first author was supported in part by NSF Grant No. 8120790 .

(C) 1988 American Mathematical Society $0002-9939 / 88 \$ 1.00+\$ .25$ per page 
2. Some preliminary results. From now on we will only be concerned with the case $m \geq 2$. Let $G F^{*}(n)$ denote the multiplicative group of $G F(n)$. Let $x$ be a generator of $G F^{*}(n)$ and for any element $y \in G F(n)$ we write $T_{x}(y)=z$ if $y=x^{z}$. An element $y \in G F^{*}(n)$ is called a desirable element if it satisfies the following three conditions:

(i) $T_{x}(y) \equiv-1(\bmod e)$.

(ii) $T_{x}(y-1) \equiv T_{x}(x-1)(\bmod 2)$.

(iii) $T_{x}(y+1) \equiv T_{x}(x+1)(\bmod 2)$.

Du and Hwang [3] proved that if $y$ is a desirable element, then the following pairing (referred to as the DH pairing in [8]) is an SSBS for $G F(n)$ :

$$
\begin{gathered}
\left(x^{i 2^{m}+2 j+1}, x^{i 2^{m}+2 j+2}\right), \quad i=0,1, \ldots, k-1 ; j=0,1, \ldots, 2^{m-2}-1, \\
\left(x^{i 2^{m}+2^{m-1}+2 j+2} y, x^{i 2^{m}+2^{m-1}+2 j+2}\right), \\
i=0,1, \ldots, k-1 ; j=0,1, \ldots, 2^{m-2}-1 .
\end{gathered}
$$

Partition the element of $G F^{*}(n)$ into $e$ residue classes $C_{t}=\left\{x^{t+i e}: i=1, \ldots\right.$, $k-1\}, t=0,1, \ldots, e-1$. Yu and Hwang [8] noticed that if for some odd $t$ the class $C_{t}$ contains two elements $u$ and $v$ such that

(a) $T(u-1) \equiv T(v-1)+1(\bmod 2)$,

(b) $T(u+1) \equiv T(v+1)+1(\bmod 2)$, and

(c) one of the two elements, say $u$, is a generator,

then $v^{-1}$ is a desirable element. They proved that except for $k=9$ and $k=3$, such a class $C_{t}$ exists for $m \geq 2$. Furthermore, for $k=9$ there exists a $C_{t}$ which contains two elements $u$ and $v$ satisfying (a) and (b).

Here, we first show that for the existence of SSBS, condition (c) is superfluous.

THEOREM 1. Suppose that for some odd the class $C_{t}$ contains two elements $u$ and $v$ such that (a) and (b) hold. Then the following pairing is an $S S B S$ for $G F(n)$ :

$$
\begin{gathered}
\left(x^{i 2^{m}+2 j+2} u, x^{i 2^{m}+2 j+2}\right), \quad i=0,1, \ldots, k-1 ; j=0,1, \ldots, 2^{m-2}-1, \\
\left(x^{i 2^{m}+2^{m-1}+2 j+2} v, x^{i 2^{m}+2^{m-1}+2 j+2}\right), \quad i=0,1, \ldots, k-1 ; j=0,1, \ldots, 2^{m-2} .
\end{gathered}
$$

ProOF. We first prove that for any $i, i^{\prime} \in\{0,1, \ldots, k-1\}$ and $j, j^{\prime} \in\{0,1, \ldots$, $\left.2^{m-2}-1\right\}, x^{i 2^{m}+2 j+2} u \neq x^{i 2^{m^{\prime}}+2^{m-1}+2 j^{\prime}+2} v$. Suppose to the contrary that

$$
x^{i 2^{m}+2 j+2} u=x^{i 2^{m^{\prime}}+2^{m-1}+2 j^{\prime}+2} v .
$$

Since $T\left(x^{i 2^{m}+2 j+2} u\right) \equiv 2 j+2+t(\bmod e)$ and

$$
T\left(x^{i 2^{m^{\prime}}+2^{m-1}+2 j^{\prime}+2} v\right) \equiv 2^{m-1}+2 j^{\prime}+2+t \quad(\bmod e),
$$

we have $2 j \equiv 2^{m-1}+2 j^{\prime}(\bmod e)$, i.e. $j-j^{\prime} \equiv 2^{m-2}(\bmod e)$. However, $\left|j-j^{\prime}\right| \leq$ $2^{m-2}-1$ and $e=2^{m}$, hence it is impossible that $j-j^{\prime} \equiv 2^{m-2}(\bmod e)$.

Now, we verify conditions (i) $-(\mathrm{v})$ in the definition of SSBS. Note that $t$ is odd. Therefore $x^{i 2^{m}+2 j+2} u$ and $x^{i 2^{m}+2^{m-1}+2 j+2} v, i=0,1, \ldots, i-1, j=0,1, \ldots, 2^{m-1}-$ 1 , run through $x^{2 s+1}, s=0,1, \ldots, 2^{m-1} k$. It follows that (i) holds. Conditions (ii) and (iii) can be easily derived from (a) and (b). Condition (iv) holds since $\left(x^{1}, x^{3}, \ldots, x^{2 p-1}\right),\left(1, x^{2}, \ldots, x^{2 p-2}\right)$ form a difference set by the Bose theorem [2]. Finally, condition (v) is satisfied since $T(-1) \equiv 2^{m-1} k \equiv 0(\bmod 2)$. 
The pairing in Theorem 1 is a generalization of the DH pairing. In fact, if $y$ is a desirable element, then $x^{-1}, y \in C_{-1}$. Let $u=x^{-1}, v=y$. Then $u$ and $v$ satisfy (a) and (b). Substituting them into Theorem 1, we can obtain the original DH pairing. As a corollary of Theorem 1, we can conclude that SSBS exists for $k=9$ (not necessarily for the DH pairing). Thus we only have the case $k=3$ left. In the following, however, we will present an approach which works for every case rather than a singular case.

A class $C_{t}$ is called a desirable class if $t$ is odd and $C_{t}$ contains two elements $u, v$ satisfying (a) and (b). Following [8], denote $O(E)$ to be the set of all odd (even) nonnegative integers less than $e$. An element $y \in G F^{*}(n)$ will be called an $(E, E)$ type if $T(y-1)$ is even and $T(y+1)$ is even. Similarly, we define the $(E, O)$ type, the $(O, E)$ type and the $(O, O)$ type. The numbers of elements in $C_{t}$ of types $(O, O),(E, E),(E, O),(O, E)$ are denoted by $N_{t}(O, O)\left(N_{t}(E, E), N_{t}(E, O)\right.$, $N_{t}(O, E)$ ). Clearly, for an odd $t C_{t}$ is desirable if and only if $N_{t}(O, O) N_{t}(E, E)+$ $N_{t}(E, O) N_{T}(O, E)>0$.

THEOREM 2. Let $t$ be odd. Then $C_{t}$ is desirable if and only if the following two conditions hold:

$\left(\mathrm{a}^{\prime}\right) C_{t}$ contains two elements $u$ and $v$ such that $T(u-1) \equiv T(v-1)+1(\bmod 2)$.

$\left(\mathrm{b}^{\prime}\right) C_{t}$ contains two elements $u^{\prime}$ and $v^{\prime}$ such that $T\left(u^{\prime}+1\right) \equiv T\left(v^{\prime}+1\right)+1$ $(\bmod 2)$.

Proof. If $C_{t}$ is desirable then $\left(\mathrm{a}^{\prime}\right)$ and $\left(\mathrm{b}^{\prime}\right)$ hold clearly. We now show that conditions $\left(\mathrm{a}^{\prime}\right)$ and $\left(\mathrm{b}^{\prime}\right)$ imply conditions (a) and (b). Suppose that $u$ and $v$, and also $u^{\prime}$ and $v^{\prime}$, do not satisfy conditions (a) and (b). Then necessarily, $T(u+1) \equiv$ $T(v+1) \equiv x(\bmod 2)$ and $T\left(u^{\prime}-1\right) \equiv T\left(v^{\prime}-1\right) \equiv v(\bmod 2)$ for some $x$ and $y$. Since $T(u-1)$ and $T(v-1)$ have different parities, one of them, say, $T(u-1)$ is congruent to $y+1(\bmod 2)$. Similarly, since $T\left(u^{\prime}+1\right)$ and $T\left(v^{\prime}+1\right)$ have different parities, one of them, say, $T\left(u^{\prime}+1\right)$ is congruent to $x+1(\bmod 2)$. Then $u$ and $u^{\prime}$ satisfy conditions (a) and (b).

3. Main results. The cyclotomic number $(s, t)$ for given $n=e k+1$ is defined as the number of elements $y \in G F^{*}(n)$ such that $T_{x}(y-1) \equiv s(\bmod e)$ and $T_{x}(y) \equiv t$ $(\bmod e)$. Define $\beta=\exp (2 \pi i / e)$ and let $J(u, v)$ be the Jacobi sum

$$
\sum_{\substack{y \in G F^{*}(n) \\ y \neq 1}} x^{u}(y) x^{v}(1-y)
$$

for a character $x$ on $G F(n)$ of order $e$. The following properties of the Jacobi sum can be found in [6]: 
LEMMA 1 .

$$
\begin{aligned}
& J(0,0)=n-2 \\
& J(0, v)=J(u, 0)=-1 \\
& J(u, v)=J(v, u) \text { has absolute value either } \sqrt{n} \text { or } 1, \\
& J(u, v)=(-1)^{u k} J(u,-u-v) \\
& J(u, v) J(-u,-v)=n \text { for } u, v \text { and } u+v \neq 0 \quad(\bmod e), \\
& J(u, v)=\sum_{s=0}^{e-1} \beta^{u s} \sum_{t=0}^{e-1} \beta^{-(u+v) t}(s, t) .
\end{aligned}
$$

It is easily verified and well known [7] that

LEMMA 2.

$$
\begin{aligned}
\sum_{t \in O}(-\beta)^{t u} & =\sum_{t \in O} \beta^{t u}=0, \quad \text { if } u \not \equiv 0, \frac{e}{2}(\bmod e) \\
& =-\frac{e}{2}, \quad \text { if } u=\frac{e}{2} \quad(\bmod e) \\
\sum_{t \in E}(-\beta)^{t u} & =\sum_{t \in E} \beta^{t u}=0, \quad \text { if } u \not \equiv 0, \frac{e}{2}(\bmod e) \\
& =\frac{e}{2}, \quad \text { if } u=\frac{e}{2} \quad(\bmod e), \\
\sum_{t=1}^{e-1} \beta^{t u} & =\sum_{t=1}^{e-1}(-\beta)^{t u}=-1, \quad \text { if } u \neq \equiv 0(\bmod e) .
\end{aligned}
$$

LEMMA 3.

$$
(s, t)=\frac{1}{e^{2}} \sum_{u=0}^{e-1} \sum_{v=0}^{e-1}(-1)^{u} \beta^{-s u-t v} J(u, v)
$$

PROOF.

$$
\begin{aligned}
(s, t) & =\frac{1}{e^{2}} \sum_{i=0}^{e-1} \sum_{j=0}^{e-1}(i, j) \sum_{u=0}^{e-1} \beta^{u(i-j-s+t)} \sum_{w=0}^{e-1} \beta^{w(t-j)} \\
& =\frac{1}{e^{2}} \sum_{u=0}^{e-1} \sum_{w=0}^{e-1} \beta^{t w-u(s-t)} \sum_{k=0}^{e-1} \beta^{u i} \sum_{k=0}^{e-1} \beta^{-(w+u) j}(i, j) \\
& =\frac{1}{e^{2}} \sum_{u=0}^{e-1} \sum_{w=0}^{e-1} \beta^{t(w+u)-u s} J(u, w) \\
& =\frac{1}{e^{2}} \sum_{u=0}^{e-1} \sum_{v=0}^{e-1} \beta^{-t v-u s} J(u,-u-v) \quad \text { setting } v=-w-u \\
& =\frac{1}{e^{2}} \sum_{u=0}^{e-1} \sum_{v=0}^{e-1} \beta^{-t v-u s}(-1)^{u k} J(u, v) \quad \text { by Lemma } 1 \\
& =\frac{1}{e^{2}} \sum_{u=0}^{e-1} \sum_{v=0}^{e-1}(-1)^{u} \beta^{-t v-u s} J(u, v) \quad \text { since } k \text { is odd. }
\end{aligned}
$$


LEMMA 4.

$$
\begin{aligned}
& \sum_{s \in O}(s, t)= \begin{cases}\left(n-1-A_{t}\right) / 2 e, & \text { if } t \neq 0, \\
\left(n-e+1-A_{t}\right) / 2 e, & \text { if } t=0,\end{cases} \\
& \sum_{s \in E}(s, t)= \begin{cases}\left(n-1+A_{t}\right) / 2 e, & \text { if } t \neq 0, \\
\left(n-e+1+A_{t}\right) / 2 e, & \text { if } t=0,\end{cases}
\end{aligned}
$$

where $A_{t}=\sum_{v=0}^{e-1} \beta^{-t v} J(e / 2, v)$.

PROOF.

$$
\begin{aligned}
\sum_{s \in O}(s, t) & =\frac{1}{e^{2}} \sum_{s \in O} \sum_{u=0}^{e-1} \sum_{v=0}^{e-1}(-1)^{u} \beta^{-s u-t v} J(u, v) \\
& =\frac{1}{e^{2}} \sum_{u=0}^{e-1} \sum_{v=0}^{e-1}(-1)^{u} \beta^{-t v} J(u, v) \sum_{s \in O} \beta^{-s u} \\
& =\frac{1}{e^{2}}\left\{\frac{e}{2} \sum_{v=1}^{e-1} \beta^{-t v} J(0, v)-\frac{e}{2} \sum_{v=0}^{e-1}(-1)^{(n-1) / 2} \beta^{-t v} J\left(\frac{e}{2}, v\right)\right\} \\
& =\frac{1}{2 e}\left\{n-2-\sum_{v=1}^{e-1} \beta^{-t v}-\sum_{v=0}^{e-1} \beta^{-t v} J\left(\frac{e}{2}, v\right)\right\} \\
& =\frac{1}{2 e}\left\{n-2-\sum_{v=1}^{e-1} \beta^{-t v}-A_{t}\right\} \\
& =\left\{\begin{array}{cc}
\left(n-1-A_{t}\right) / 2 e, & \text { if } t \neq 0, \\
\left(n-e+1-A_{t}\right) / 2 e, & \text { if } t=0 .
\end{array}\right.
\end{aligned}
$$

Similarly, we can verify the other equation.

LEMMA 5. $\sum_{t=0}^{e-1} A_{t}^{2}=e^{2}(k e-2 k+1)$.

PROOF.

$$
\begin{aligned}
\sum_{t \in O} A_{t}^{2} & =\sum_{t \in O} \sum_{u=0}^{e-1} \sum_{v=0}^{e-1} J\left(\frac{e}{2}, u\right) J\left(\frac{e}{2}, v\right) \beta^{-t(u+v)} \\
& =\sum_{u=0}^{e-1} J\left(\frac{e}{2}, u\right) \sum_{v=0}^{e-1} J\left(\frac{e}{2}, v\right) \sum_{t \in O} \beta^{-t(u+v)} \\
& =\sum_{u=0}^{e-1} J\left(\frac{e}{2}, u\right)\left\{J\left(\frac{e}{2},-u\right) \cdot \frac{e}{2}-J\left(\frac{e}{2}, \frac{e}{2}-u\right) \frac{e}{2}\right\} \\
& =\frac{e}{2}\left\{(e-2) n+2-\sum_{u=0}^{e-1} J\left(\frac{e}{2}, u\right)^{2}\right\}
\end{aligned}
$$

since

and

$$
J\left(\frac{e}{2}, \frac{e}{2}-u\right)=(-1)^{(e / 2) k} J\left(\frac{e}{2}, \frac{e}{2}-\left(\frac{e}{2}-u\right)\right)=J\left(\frac{e}{2}, u\right)
$$

$$
J\left(\frac{e}{2}, u\right) J\left(\frac{e}{2},-u\right)= \begin{cases}n, & \text { if } u \neq 0, e / 2 \\ 1, & \text { if } u=0, e / 2\end{cases}
$$


Similarly,

$$
\sum_{t \in E} A_{t}^{2}=\frac{e}{2}\left\{(e-2) n+2+\sum_{u=0}^{e-1} J\left(\frac{e}{2}, u\right)^{2}\right\}
$$

Therefore,

$$
\begin{aligned}
\sum_{t=0}^{e-1} A_{t}^{2} & =e((e-2) n+2)=e((e-2)(k e+1)+2) \\
& =e^{2}(k e-2 k+1) .
\end{aligned}
$$

LEMMA 6 .

$$
\sum_{t=1}^{e-1}\left(\sum_{s \in O}(s, t)\right)\left(\sum_{s \in E}(s, t)\right) \geq \frac{1}{4}\left(k(k-1) e-(k-1)^{2}\right) .
$$

ProOF. By Lemma 3 and Lemma 4, we have

$$
\begin{gathered}
\sum_{t=1}^{e-1}\left(\sum_{s \in O}(s, t)\right)\left(\sum_{s \in E}(s, t)\right)=\sum_{t=1}^{e-1} \frac{1}{4 e^{2}}\left((n-1)^{2}-A_{t}^{2}\right) \\
=\frac{1}{4 e^{2}}\left((e-1)(n-1)^{2}-\sum_{t=1}^{e-1} A_{t}^{2}\right) \\
\geq \frac{1}{4 e^{2}}\left((e-1)(n-1)^{2}-e^{2}(k e-2 k+1)\right)
\end{gathered}
$$

(by Lemma $3, A_{0}$ is a real number, so $A_{0}^{2} \geq 0$ )

$$
\begin{aligned}
& =\frac{1}{4 e^{2}}\left((e-1)(k e)^{2}-e^{2}(k e-2 k+1)\right) \\
& =\frac{1}{4}\left(k(k-1) e-(k-1)^{2}\right) .
\end{aligned}
$$

Let $P$ denote the number of odd $t$ satisfying $\left(\mathrm{a}^{\prime}\right)$.

LEMMA 7. $P \geq e / 4+1$ for $k \geq 3$.

PROOF. Note that $\sum_{s \in O}(s, t)$ and $\sum_{s \in E}(s, t)$ are two nonnegative integers such that $\sum_{s \in O}(s, t)+\sum_{s \in E}(s, t)=k$. Since $k$ is odd, we have

$$
\left(\sum_{s \in O}(s, t)\right)\left(\sum_{s \in E}(s, t)\right) \leq \frac{k+1}{2} \cdot \frac{k-1}{2}=\frac{1}{4}\left(k^{2}-1\right) .
$$

For any $t \in\{1,2, \ldots, e-1\}$ condition $\left(\mathrm{a}^{\prime}\right)$ does not hold if and only if

$$
\left(\sum_{s \in O}(s, t)\right)\left(\sum_{s \in E}(s, t)\right)=0 \text {. }
$$

Thus, we obtain

$$
\sum_{t=1}^{e-1}\left(\sum_{s \in O}(s, t)\right)\left(\sum_{s \in E}(s, t)\right) \leq\left(P+\frac{e}{2}-1\right) \cdot \frac{1}{4}\left(k^{2}-1\right) .
$$


By Lemma 5, we have

$$
(P+e / 2-1)\left(k^{2}-1\right) \geq k(k-1) e-(k-1)^{2} .
$$

Therefore,

$$
\begin{aligned}
P & \geq \frac{k}{k+1} e-\frac{k-1}{k+1}-\frac{e}{2}+1 \\
& \geq \frac{3}{4} e-\frac{e}{2}+\frac{2}{k+1}=\frac{e}{4}+\frac{2}{k+1} .
\end{aligned}
$$

Since $P$ is an integer, $P \geq e / 4+1$.

LEMMA 8. There exists a desirable class for $k \geq 3$ and $m \geq 2$.

PROOF. Let $Q$ denote the number of odd $t$ satisfying $\left(\mathrm{b}^{\prime}\right)$. We claim that $P=Q$. To see this, it suffices to prove that $t$ satisfies $\left(\mathrm{a}^{\prime}\right)$ if and only if $e / 2+t$ satisfies $\left(\mathrm{b}^{\prime}\right)$. We verify it as follows.

$$
\begin{aligned}
& T(u-1) \equiv T(v-1)+1 \quad(\bmod 2) \\
& \quad \Leftrightarrow T(-u+1) \equiv T(-v+1)+1 \quad(\bmod 2) \\
& \quad \Leftrightarrow T\left(x^{e k / 2} u+1\right) \equiv T\left(x^{e k / 2} v+1\right) \quad(\bmod 2) .
\end{aligned}
$$

Clearly, $u, v \in C_{t}$ if and only if $x^{e k / 2} u, x^{e k / 2} v \in C_{e k / 2+t}=C_{e / 2+t}$. This concludes our claim. Now, we have $P+Q \geq 2(e / 4+1)=e / 2+2$. Hence, there exists an odd $t$ satisfying both $\left(\mathrm{a}^{\prime}\right)$ and $\left(\mathrm{b}^{\prime}\right)$.

THEOREM 3. SSBS exists for all $k \geq 3$ and $m \geq 2$.

ProOF. By Lemma 8 and Theorem 1.

4. Some concluding remarks. Our approach also proves the conjecture of $\mathrm{Yu}$ and Hwang [7]. It suffices to prove that for $k=3$ and 9 there exist two elements $u$ and $v$ satisfying conditions (a), (b), (c). Note that for $k=3$ every $C_{t}$ for an odd $t$ contains 2 generators. Therefore, at least one of the two elements in a desirable class is a generator. For $k=9$ every $C_{t}$ for odd $t$ contains 6 generators. If $C_{t}$ does not contain two elements $u, v$ satisfying (a), (b) and (c), then one of the following cases occurs:

(1) $N_{t}(O, O) N_{t}(E, E)+N_{t}(O, E) N_{t}(E, O)=0$.

(2) $N_{t}(O, O) N_{t}(E, E) \neq 0 ; N_{t}(O, E) N_{t}(E, O)=0$, and there is no generator of $(O, O)$ type or $(E, E)$ type in $C_{t}$.

(3) $N_{t}(O, O) N_{t}(E, E)=0, N_{t}(O, E) N_{t}(E, O) \neq 0$, and there is no generator of $(O, E)$ type or $(E, O)$ type in $C_{t}$.

Lemma 7 rejects the possibility of (1). If (2) occurs, then $N_{t}(O, O)+N_{t}(E, E)=3$, so

$$
N_{t}(O, O) N_{t}(E, E)+N_{t}(O, E) N_{t}(E, O) \leq 2 .
$$

Similarly, if (3) occurs then the above inequality again holds. Thus we have

$$
\sum_{t \in O}\left\{N_{t}(O, O) N_{t}(E, E)+N_{t}(O, E) N_{t}(E, O)\right\} \leq e .
$$


However, Yu and Hwang [8] have proved

$$
\sum_{t \in O}\left\{N_{t}(O, O) N_{t}(E, E)+N_{t}(O, E) N_{t}(E, O)\right\} \geq \frac{1}{16} k(k-4) e>e
$$

for $k=9$, a contradiction.

Finally we show that no desirable element can exist for $k=1$. Note that for $k=1$ each of the $e$ cyclotomic classes contains only one element. Therefore the only element which satisfies condition (i): $T_{x}(y)=-1(\bmod e)$ is $y=x^{-1}$. But

$$
x-1=-x\left(x^{-1}-1\right)=x^{e / 2+1}\left(x^{-1}-1\right) \text {. }
$$

Therefore

$$
T_{x}\left(x^{-1}-1\right) \not \equiv T_{x}(x-1),
$$

i.e., condition (ii) of a desirable element is violated.

Of course the above proof only shows that the $\mathrm{DH}$ pairing does not work for $k=1$. The nonexistence of SSBS for $k=1$ remains an open conjecture [5]. However, when conditions (iii) and (v) of SSBS are waived (an SSBS then becomes a balanced starter), then Hanner [4] proved that balanced starters exist for all odd prime powers.

\section{REFERENCES}

1. E. R. Berlekamp and F. K. Hwang, Contributions for balanced Howell rotations for bridge tournaments, J. Combin. Theory 12 (1972), 159-166.

2. R. C. Bose, On a resolvable series of balanced incomplete block designs, Sankhya 8 (1947), 249-256.

3. D.-Z. Du and F. K. Hwang, Symmetric skew balanced starters and complete balanced Howell rotations, Trans. Amer. Math. Soc. 271 (1982), 409-413.

4. O. Hanner, Construction of balanced Howell rotations for $2(p+1)$ partnerships, J. Combin. Theory A33 (1982), 205-212.

5. F. K. Hwang, Starters, balanced starters and partitionable starters, Bull. Inst. Math. Acad. Sinica 11 (1983), 561-572.

6. T. Storer, Cyclotomy and difference sets, Markham, Chicago, Ill., 1967.

7. J. E. Yu and F. K. Hwang, Symmetric skew balanced starters for odd prime powers, Ars Combinatoria 19 (1985), 187-192.

8. _ The existence of symmetric skew balanced starters for odd prime powers, European $\mathrm{J}$. Combin. 9 (1988), 153-160.

MAThematical SCiences Research Institute, Berkeley, California 94720

AT\&T BELl Laboratories, MURRAy Hill, NEW JERSEy 07974

D. Z. Du, institute of Applied Mathematics, ACademia Sinica, Beijing, China 